\title{
A Stage-Based Mobile Intervention for Substance Use Disorders in Primary Care
}

Deborah Levesque, PhD; Cindy Umanzor, MPH; Emma de Aguiar, BA

Pro-Change Behavior Systems, Inc, South Kingstown, RI, United States

Corresponding Author:

Deborah Levesque, $\mathrm{PhD}$

Pro-Change Behavior Systems, Inc

1174 Kingstown Road

Suite 101

South Kingstown, RI, 02879

United States

Phone: 14013602975

Fax: 14013602975

Email: dlevesque@prochange.com

\section{Abstract}

Background: Over 20 million American adults meet diagnostic criteria for a substance use disorder (SUD). However, only $10-11 \%$ of individuals requiring SUD treatment receive it. Given their access to patients, primary care providers are in a unique position to perform universal screening, brief intervention, and referral to treatment (SBIRT) to fill gaps in services and make referrals to specialty treatment when indicated. While SBIRT has shown success in reducing tobacco and non-dependent alcohol use, the data on SBIRT for dependent alcohol use and illicit drug use are less promising. Major barriers to SBIRT include limited time and resources for SBIRT among providers and low motivation to change among many patients.

Objective: The objective of this research was to develop and test the acceptability of a prototype of a mobile-delivered substance use risk intervention (SURI) for primary care patients and a clinical dashboard for providers that can address major barriers to SBIRT for risky drug use. To reduce provider burden, SURI delivers universal screening and feedback on SUD risk via mobile tools to patients at home or in the waiting room. For patients at risk, it also delivers a brief intervention based on the Transtheoretical Model of Change to facilitate progress through the stages of change for quitting the most problematic drug and for seeking treatment if indicated. The prototype also delivers 30 days of stage-matched text messages and four online activities addressing key topics (eg, managing cravings). For providers, the clinical dashboard summarizes the patient's SUD risk scores and stage of change data and provides stage-matched scripts to guide in-person sessions.

Methods: Feasibility test participants were 3 providers at a federally qualified health center and 5 of their patients with a known SUD. Providers completed a 45-minute webinar training session on the SURI tools, delivered dashboard-guided SBIRT session(s), and completed a brief acceptability survey. Patients completed an online SURI session and in-person SBIRT session, accessed other program components, and completed 3 acceptability surveys over 30 days. Questions in the surveys were adapted from the National Cancer Institute's Education Materials Review Form. Response options ranged from 1=strongly disagree to 5=strongly agree. The criterion for establishing feasibility was an overall rating of 4 or higher across items.

Results: For providers, mean acceptability ratings ranged from 3.7 to 5.0, with an overall mean rating of 4.3. Notably, all providers gave a rating of 5.0 for the item "The program can give me helpful information about my patient." One patient dropped out of the study before accessing any study materials. For the remaining patients, mean acceptability ratings for the mobile- and provider-delivered SBIRT session ranged from 4.0 to 5.0, with an overall mean rating of 4.5; mean ratings of the follow-up text messages and online activities ranged from 3.6 to 4.8 , with an overall rating of 4.0. One of the most highly rated items was "The program could help me make some positive changes" (4.5).

Conclusions: The SURI program and clinical dashboard, developed to reduce barriers to SBIRT in primary care, were well received by care providers and patients.

(iproc 2016;2(1):e30) doi: 10.2196/iproc.6157 


\section{KEYWORDS}

screening, brief intervention, and referral to treatment (SBIRT); primary care; mobile intervention; Transtheoretical Model of Change

This poster was presented at the Connected Health Symposium 2016, October 20-21, Boston, MA, United States. The poster is displayed as an image in Figure 1 and as a PDF in Multimedia Appendix 1.

Figure 1. Poster.

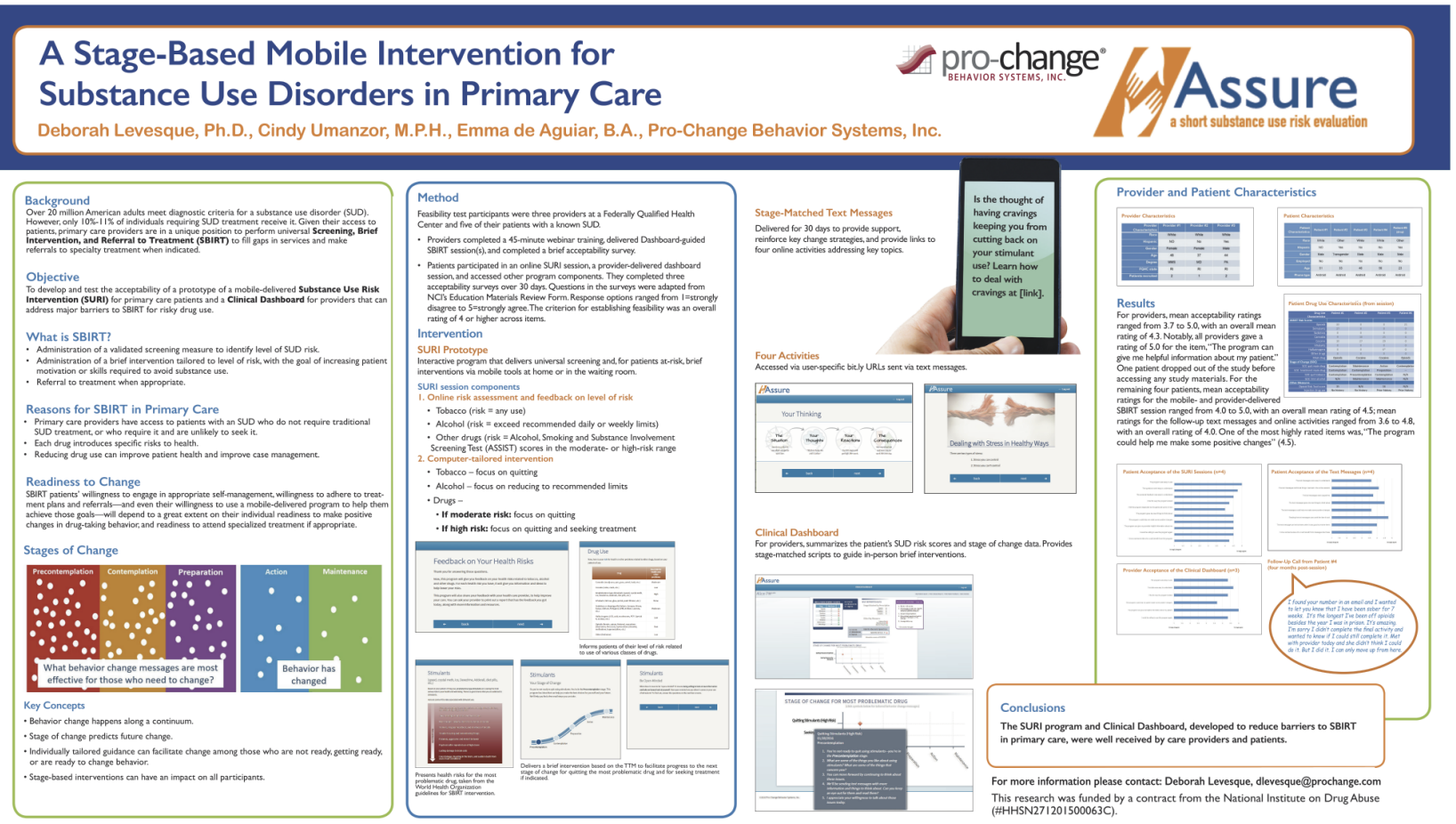

\section{Multimedia Appendix 1}

Poster.

[PDF File (Adobe PDF File), 3MB-Multimedia Appendix 1]

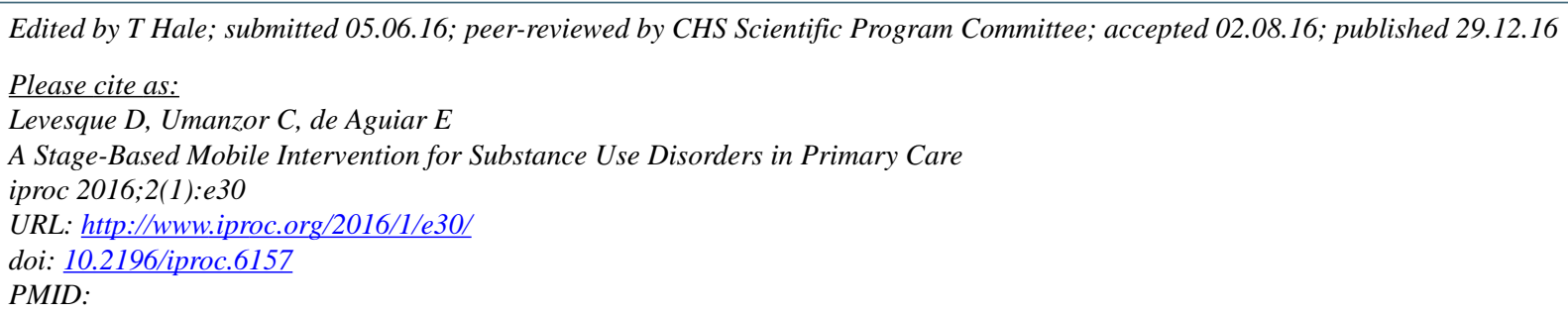

(CDeborah Levesque, Cindy Umanzor, Emma de Aguiar. Originally published in Iproceedings (http://www.iproc.org), 29.12.2016. This is an open-access article distributed under the terms of the Creative Commons Attribution License (http://creativecommons.org/licenses/by/2.0/), which permits unrestricted use, distribution, and reproduction in any medium, provided the original work, first published in Iproceedings, is properly cited. The complete bibliographic information, a link to the original publication on http://www.iproc.org/, as well as this copyright and license information must be included. 